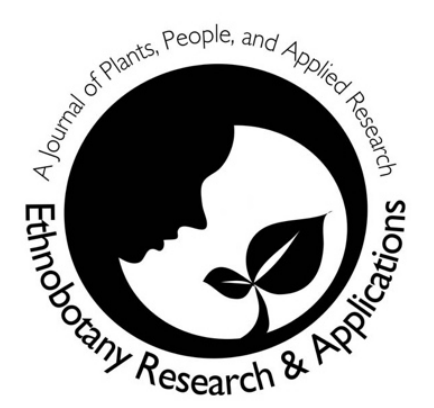

\title{
Edible Mushrooms of Ecuador: consumption, myths and implications for conservation
}

Paúl Gamboa-Trujillo, Felipe Wartchow, Carlos CerónMartinez, Domingo Andi, Pedro Uwinjin, Gladys Grefa, Maria Entza, Eulalia Chimbo, Julio Chimbo, Joaquina Payaguaje, Norma Piyaguaje, Delfin Payaguaje, Vicente Licuy, Venancio López, Marcelo Mendua, Mauricio Criollo, María Jati, Simón De La Cruz, Manuel Calazacón, Segundo Flores, Esteban Aules, Carmen Aigaje, María De Aro, Amparo Morales, Yolanda Murillo, German Farinango and Tatiana B. Gibertoni

\section{Research}

\begin{abstract}
Background: Ecuador is divided into three geographical regions: Amazon, Coast and Mountains, on which one having its own empirical knowledge and traditions in relation to their natural environments. Studies about traditional knowledge of macrofungi were initially carried out in the Brazilian Amazon and later in Colombia, Guyana and Venezuela. In Ecuador few studies have been published. Thus, the aim of this study is to investigate many ethnomycological data of the edible mushrooms used by several ethnic Ecuadorian groups, discussing the meanings of species' vernacular names and related myths.
\end{abstract}

\section{Correspondence}

Paúl Gamboa-Trujillo ${ }^{1,2,3,4 *}$, Felipe Wartchow ${ }^{5 *}$, Carlos Cerón-Martinez ${ }^{3}$, Domingo Andi ${ }^{6}$, Pedro Uwinjin $^{6}$, Gladys Grefa ${ }^{6}$, Maria Entza ${ }^{6}$, Eulalia Chimbo $^{6}$, Julio Chimbo ${ }^{6}$, Joaquina Payaguaje ${ }^{6}$, Norma Piyaguaje ${ }^{6}$, Delfin Payaguaje ${ }^{6}$, Vicente Licuy $^{6}$, Venancio López ${ }^{6}$, Marcelo Mendua ${ }^{6}$, Mauricio Criollo ${ }^{6}$, María Jati ${ }^{6}$, Simón De La Cruz ${ }^{6}$, Manuel Calazacón ${ }^{6}$, Segundo Flores ${ }^{6}$, Esteban Aules $^{6}$, Carmen Aigaje ${ }^{6}$, María De Aro ${ }^{7}$, Amparo Morales $^{7}$, Yolanda Murillo ${ }^{7}$, German Farinango ${ }^{7}$ and Tatiana B. Gibertoni ${ }^{4 *}$
1Facultad de Ciencias Biológicas-Carrera de
Ciencias Biológicas Universidad Central del Ecuador
-UCE, Ecuador.
${ }^{2}$ Laboratorio de Micología Aplicada, Facultad de
Ingeniería Química-UCE, Ecuador.
${ }^{3}$ Herbario Alfredo Paredes QAP-UCE- Sección
Micológica,Avenida América y Gato Sobral,
Ciudadela Universitaria, Ecuador.
${ }^{4}$ Universidade Federal de Pernambuco,
Departamento de Micologia/CCB, Av. Prof. Nelson
Chaves, s/n', CEP: 50670-901, Recife, PE, Brazil.
${ }^{5}$ Universidade Federal da Paraíba, Departamento de
Sistemática e Ecologia/CCEN, CEP: 58051-970,
João Pessoa, PB, Brazil.
6 Directivos y profesores Asociación de Comunidad
Indígena de la Amazonia, ACIA, Puyo, Pastaza,
Esmeraldas, Santo Domingo de los Tsachilas,
Comunidad Shuar de Wapu- CONAIE- ECUADOR
Av. Granados E10-275 y 6 de Diciembre Quito,
Pichincha, Ecuador.
${ }^{7}$ Directivos de la Asociación de Caficultores Río
Intag, Parroquia Cuellaje, Imbabura, Ecuador.
Corresponding author:
P. Gamboa-Trujillo - jpgamboa@uce.edu.ec/
paulgamboativi@hotmail.com
*PGT, FW and TBG are the lead authors
18:38 (2019) Research \& Applications

Methods: The ethnomycological data of the edible mushrooms were collected in 13 indigenous 
communities of Ecuador during periods of high rainfall from 2002 to 2016, with interviews and forums. Additionally, the fungal specimens were collected, and voucher deposited at Herbarium QAP.

Results: Thirty-seven edible species of macrofungi were identified and some of them are discussed in relation to aspects like local peoples' mythologies, habits or tradition, their preparation for consumption and myths. Thus, this research added new several ethnomycological data and helped to understand the mycophagous behavior in Ecuador.

Conclusions: These studies should continue in order to prevent ethnobiological loss caused by the degradation of the environment in which these people live, causing a loss of the biodiversity. In addition, biological conservation of these ecosystems is urgent since many natural resources are used for these indigenous communities as food consumptions.

Key words: Amazonia, biodiversity, Coast, Fungi, Páramos, South America

\section{Background}

Ecuador is divided into three geographical regions: Amazon (Amazonía), characterized by evergreen forest flooded by white waters; Coast (Costa), characterized by lowlands and evergreen mountains; and Mountains (Sierra), covered either by xerophytic vegetation, cloud forest, or 'páramos' in the higher altitudes (Sierra 1999). In these regions, there are 13 indigenous groups, each one having its own empirical knowledge and traditions in relation to their natural environments (Gamboa-Trujillo 2005, Gamboa-Trujillo et al. 2005).

Regarding ethomycological studies, they have been intensified in other Latin-American countries such as Mexico (Montoya et al. 2003, 2004, 2008, GaribayOrijel et al. 2006, 2007, Ruan-Soto et al. 2006, Pérez-Moreno et al. 2008) and Guatemala (Morales et al. 2006), in Central and North America.

In South America, studies about traditional knowledge of macrofungi were initially carried out in the Brazilian Amazon with the Yanomami (Prance 1973) and later by Fidalgo and Prance (1976) with the Samana. Previous studies by Fidalgo (1965, 1985 ) already suggested that the mycophilous habits among the Brazilian indigenous people were lost during the Portuguese invasion. Later, Henkel et al. (2004) discussed about some edible mushrooms used by the Patamona and Henkel (2005) investigated about an alcoholic beverage resulted from cassava fermentation by Rhizopus sp. in Guyana. In Venezuela Zent et al. (2004) and Zent (2008) discussed the knowledge, useful applications and mythological relevance of several fungal species by Amazonian and Guiana people. Other relevant study was performed by Vasco-Palacios et al. (2008) who showed an ecological knowledge given by various indigenous groups from Medio Caquetá, Colombia. More recently, Cardoso et al. (2010) drew parallels between the fungal nomenclature of Brazilian people with classical taxonomy and phylogenetic systematic and concluded that some names are similar to current proposed phylogenetic classifications. In Ecuador, few studies have been published. Gamboa-Trujillo (2005) and GamboaTrujillo et al. (2005) performed a more introductory study about enthomycology in this country. Finally, a descriptive article was recently given by GamboaTrujillo et al. (2014) during investigations on the edibility of Gymnopus nubicola Halling in the páramos

Thus, the aim of this study is to investigate the ethnomycological data of the edible mushrooms used by several ethnic Ecuadorian groups, discussing the meanings of species' vernacular names, forms of consumption, and related myths.

\section{Materials and Methods}

Thirteen communities were visited during winter (rainy) season between September and February 2002 to 2016, inside and around natural reserves, and public markets. These are Chachi/ Chafi'ki (Coastal region), Tsachila/ Tsafi'ki / (Coastal region), Mestizos/ Spanish with Kichwa (Mountainous area), Kichwas/Kichwa (Amazonia), Kichwas/Kichwa (Highlands), Secoyas/ Pai'coca (Amazonia), Sionas/ Bai'coca (Amazonia), Shuar/ Shuar Chicham (Amazonia).

In this study, only macrofungi belonging to the phyla Basidiomycota and Ascomycota (Kirk et al. 2008) were collected or bought in popular markets in the mountains and Amazon region when it had been reported that they are of some kind of use. During and after harvesting, the community members, especially healers and wise men and women between 40 and 90 years old, were invited to participate in informal interviews (without payment) and informal open forums mediated by teachers. During the interviews and forums, data on use, vernacular names in local language, etymology and form of consumption of the fungi were obtained. The groups ranged from one to eight persons, who accepted to participate voluntarily. 
For taxonomic studies of the macrofungi, the usual methodologies were used (e.g. Singer 1986, Ryvarden 1991). For identification of species, the dichotomous keys of Corner (1950), Dennis (1956), Heinemann (1961), Ryvarden (1991), Guzmán (1997), Iturriaga and Pfister (2006) among others were used. The fungal materials were deposited at the Herbaria QAP and URM (Thiers, continuously updated).

\section{Results and Discussion \\ Demographic data}

The majority of the native communities in the different regions of the Continental Ecuador are dedicated to agriculture, plant and fungi collection, but also hunting and fishing. Some of these groups are very numerous, as for example the Amazonians Kichwas and other are in danger of disappearance as occurred with the Zapara community.

\section{General remarks on edible species}

In this study, 13 ethnic groups were investigated, on which only two, the Epera and the Afroecuadorian communities, did not present mycophagous activity, but they provided some mycophilous information. The other 11 communities revealed at least 90 records of edible mushrooms among 37 species and 32 vernacular names (Table 1).

In the communities, the women generally harvest the mushrooms, but in some towns, it is a family activity, in which all members participate. They put the macrofungi in plastic bags and natural fibers baskets. In addition to this activity, they gather medicinal herbs, wild fruits such as Rubus bogotense Kunth (mora), Passiflora mixta L. (taxo), Solanum caripense Dunal (tzimbalos) and straw of Calamagrostis spp., used to prepare nests for chicken, Cavia aperea Erxleben (guinea pig or cuy) and other animals.

\section{Vernacular Names}

The studied communities have mono-, bi- and trivernacular names for edible macrofungi. They are related to animals, color, form, myths, odor, and plants, among others. The suffixes ala, tëti, esemb, kallamba, kallumpa, katsapija, kijte, kijtiutiu and tsina are used for designation of the name 'mushrooms'. We can cite here some examples:Favolus tenuiculus (Fig. 1A) is named busum ala due to its hymenophore with hexagonal pores similar to the inner surface of the cow's stomach (busum = cow stomach). This species is also calling anj kijtiutiu due to its whitish color (anj = white). Volvariella volvacea (Fig. 1B) is named "kijtiutiu pandachi" because it usually grows on decaying banana leaves (pandachi = banana leaves), while Auricularia delicate (Fig. 1.C) is named iwianchi kuishi, the devil's ear (iwianchi = devil; kuishi = ear). Cotylidia aurantiaca (is called aya ala by the Kichwa community, which can be translated as 'Ghost's mushroom' (aya = ghost), while Agaricus argyropotamicus and $A$. pampeanus are named kallamba de finados, due to its consumption during the Day of the Dead.

\section{Mushroom Consumption}

In most ethnic groups, the stipe's base of the mushrooms is cut off, because it contains substrate debris, or have cartilaginous consistence. However, some people use the entire mushroom to cook, smoke or fry. Below we give some example of mushrooms and their form of consumption. All of them are eaten after washed (Fig. 2) then boiled or roasted.

Some mushrooms, such as Auricularia delicata, $A$. fuscosuccinia, Favolus tenuiculus, Pleurotus concavus and $P$. djamor, among others, are consumed in the Coast and Amazon regions generally cooked in boiling water or wrapped in leaves of Heliconia stricta (Huber) Griggs (platanillo), which are placed over fire in a rudimentary handmade stove until they are roasted (Fig. 3A-I). This dish has several names:mayto by the Amazon Kichwas; ayampaco by the Shuars and Shiwiars; cuaisu'u by the Secoyas and Sionas; and pando by the Chachis. In the Sierra region, those mushrooms are fried or prepared in a thick soup with chicken, fish or wild rabbit.

Auricularia fuscosuccinea was also reported as edible by Ruán-Soto et al. (2006) in Mexico. In Ecuador, the Shuar children also play with this species. The basidiome of $A$. fuscosuccinea can be separate in layers very easily; even so, they make a hole in the center and fill air inside with their mouths, using the mushroom as small balloon (Fig. 4A-B) The child that makes the loudest sound when the balloon bursts is the winner. Ruan-Soto et al. (2006) reported $A$. delicata and $A$. polytricha that are used by children in the same way, but according to our observations, these species do not have the separable membranes as $A$. fuscosuccinea. Interesting to note that other unidentified taxa of Auricularia are used as food by the Sanama in Brazil (Fidalgo and Prance 1976), the Temuans in Malaysia (Chang and Lee 2004) and Nepalese people (Adhikari et al. 2005). 
Ethnobotany Research and Applications

\begin{tabular}{|c|c|c|c|c|c|}
\hline Community/Language/ Region & Scientific name & Vernacular name & Etymology & Substrate & $\begin{array}{c}\text { Selling at } \\
\text { market }\end{array}$ \\
\hline \multirow[t]{7}{*}{ Chachi/ Chafi'ki / Coast } & $\begin{array}{l}\text { Auricularia delicata (Mont.) } \\
\text { Henn. }\end{array}$ & Ishk kijtiutiu & Edible mushroom & Wood & No \\
\hline & Favolus tenuiculus P.Beauv. & Anj kijtiutiu & White, edible mushroom & Wood & No \\
\hline & $\begin{array}{l}\text { Oudemasiella cubensis (Berk. \& } \\
\text { M.A. Curtis) R.H. Petersen }\end{array}$ & Anj kijtiutiu & White, edible mushroom & Wood & No \\
\hline & Phillipsia domingensis Berk. & Pachi kijtiutiu & Edible mushroom & Wood & No \\
\hline & $\begin{array}{l}\text { Pleurotus concavus (Berk.) } \\
\text { Singer }\end{array}$ & Anj kijtiutiu & White, edible mushroom & Wood & No \\
\hline & $\begin{array}{l}\text { Pleurotus djamor (Rumph. ex } \\
\text { Fr.) Boedijn }\end{array}$ & Anj kijtiutiu & White, edible mushroom & Wood & No \\
\hline & $\begin{array}{l}\text { Volvariella volvacea (Bull.) } \\
\text { Singer }\end{array}$ & $\begin{array}{l}\text { Kijtiutiu pandachi } \\
\text { Ishk kijtiutiu } \\
\text { Bulun kijtiutiu }\end{array}$ & $\begin{array}{l}\text { Banana's leaves } \\
\text { Edible mushroom } \\
\text { Reddish mushroom, edible }\end{array}$ & $\begin{array}{l}\text { Banana's } \\
\text { leaves }\end{array}$ & No \\
\hline \multirow{6}{*}{ Tsachila/ Tsafi'ki / Coast } & Favolus tenuiculus P.Beauv. & Kijte pa & Edible mushroom & Wood & No \\
\hline & Lentinus scleropus (Pers.) Fr. & Chide kijte & Tree's mushroom & Wood & No \\
\hline & Lentinus velutinus Fr. & Pavan kijte & Black mushroom & Wood & No \\
\hline & Monilia sp. & Lopo & Mushroom for fermentation & Cassava & No \\
\hline & Polyporus tricholoma Mont. & Dodo kijte & Tree's mushroom & Wood & No \\
\hline & $\begin{array}{l}\text { Tetrapyrgos alba (Berk. \& M.A. } \\
\text { Curtis) E. Horak }\end{array}$ & Na kijte & Mushroom for children & Wood/litter & No \\
\hline \multirow{7}{*}{$\begin{array}{l}\text { Mestizos/ Spanish with } \\
\text { Kichwa/Mountainous area }\end{array}$} & Agaricus argyropotamicus Speg. & Kallamba de comer & Edible mushroom & Soil & Yes \\
\hline & Agaricus pampeanus Speg. & Kallamba & Mushroom & Soil & Yes \\
\hline & $\begin{array}{l}\text { Coprinus comatus var.comatus } \\
\text { (O.F.Müll.) Pers. }\end{array}$ & Kallamba de comer & Edible mushroom & Soil & No \\
\hline & Favolus tenuiculus P.Beauv. & Kallumpa & Edible mushroom & Wood & No \\
\hline & Suillus luteus (L.)Roussel & Kallamba del pino & Pine's mushroom & Soil & Yes \\
\hline & $\begin{array}{l}\text { Pleurotus djamor (Rumph.ex } \\
\text { Fr.) Boedjin }\end{array}$ & Española & White Spanish woman & Wood & No \\
\hline & $\begin{array}{l}\text { Auricularia fuscosuccinea } \\
\text { (Mont.) Henn. }\end{array}$ & Oreja de mono & Monkey's ear & Wood & No \\
\hline Kichwas/Kichwa/Highland & Agaricus pampeanus Speg. & $\begin{array}{l}\text { Kallamba de } \\
\text { finados }\end{array}$ & $\begin{array}{l}\text { Day of the Dead's } \\
\text { mushroom }\end{array}$ & Soil & Yes \\
\hline
\end{tabular}

Published: 12 December 2019

http://dx.doi.org/10.32859/era.18.38.1-15 
Ethnobotany Research and Applications

\section{Kichwas/Kichwa/Amazonia}

\begin{tabular}{|c|c|c|c|c|}
\hline & Carne de Diosito & Meat of God & & \\
\hline $\begin{array}{l}\text { Auricularia delicata (Mont.) } \\
\text { Henn. }\end{array}$ & Oreja de mono & Monkey's ear & Wood & No \\
\hline $\begin{array}{l}\text { Auricularia fuscosuccinea } \\
\text { (Mont.) Henn. }\end{array}$ & Oreja de mono & Monkey's ear & Wood & \\
\hline Gymnopus nubicola Halling & Kallamba & Mushroom & Soil & Yes \\
\hline Favolus tenuiculus P.Beauv. & Pusum o Pusunera & Cow's stomach & Wood & No \\
\hline $\begin{array}{l}\text { Pleurotus djamor (Rumph. ex } \\
\text { Fr.) Boedijn }\end{array}$ & Española blanca & White Spanish woman & Wood & No \\
\hline Suillus luteus (L.) Roussel & Kallamba & Mushroom & Soil & Yes \\
\hline Auricularia cornea Ehrenb. & Calulu ala & Jelly mushroom & Wood & No \\
\hline $\begin{array}{l}\text { Auricularia delicata (Mont.) } \\
\text { Henn. }\end{array}$ & Calulu ala & Jelly mushroom & Wood & No \\
\hline $\begin{array}{l}\text { Auricularia fuscosuccinea } \\
\text { (Mont.) Henn. }\end{array}$ & Calug calug ala & Jelly mushroom & Wood & No \\
\hline $\begin{array}{l}\text { Ophiocordyceps melolonthae } \\
\text { (Tul. \& C. Tul.) G.H. Sung, J.M. } \\
\text { Sung, Hywel-Jones \& Stapafora }\end{array}$ & Garauto ala & Worm's mushroom & Beetle & No \\
\hline $\begin{array}{l}\text { Coriolopsis floccosa (Jungh.) } \\
\text { Ryvarden }\end{array}$ & Chonta ala & Chonta's mushroom & Wood & No \\
\hline $\begin{array}{l}\text { Cotylidia aurantiaca (Pat.) A.L. } \\
\text { Welden }\end{array}$ & Aya ala & Ghost's mushroom & Wood & No \\
\hline $\begin{array}{l}\text { Dacryopinax spathularia } \\
\text { (Schwein.) G.W. Martin }\end{array}$ & Calulu ala & Jelly mushroom & Wood & No \\
\hline Favolus tenuiculus P.Beauv. & $\begin{array}{l}\text { Pusum/chincha ala } \\
\text { Yurac ala } \\
\text { Ñuto ala } \\
\text { Api ala } \\
\text { Caspi ringri ala }\end{array}$ & $\begin{array}{l}\text { Cow stomach mushroom } \\
\text { Trail's mushroom } \\
\text { Soft mushroom } \\
\text { Bee's mushroom } \\
\text { Stick's ear mushroom }\end{array}$ & Wood & No \\
\hline Gymnopilus lepidotus Hesler & Chonta ala & Chinta's mushroom & Wood & No \\
\hline Hygrocybe sp. & Rinri ala & Ear's mushroom & Soil & No \\
\hline Lentinus crinitus (L.) Fr. & IIma ala & Soft mushroom & Wood & No \\
\hline Lentinus sajor-caju (Fr.) Fr. & Taka ala & Trunk's mushroom & Wood & No \\
\hline Leucocoprinus sp. & $\begin{array}{l}\text { Chonta ala } \\
\text { Chincha ala }\end{array}$ & $\begin{array}{l}\text { Chonta's mushroom } \\
\text { Edible mushroom }\end{array}$ & Soil & No \\
\hline
\end{tabular}

Published: 12 December 2019

http://dx.doi.org/10.32859/era.18.38.1-15 
Ethnobotany Research and Applications

\begin{tabular}{|c|c|c|c|c|c|}
\hline & Marasmiellus sp. & Shiquitu ala & Tiny mushroom & Litter/Wood & No \\
\hline & Marasmius cladophyllus Berk. & Quillu ala & Yellow mushroom & Litter & No \\
\hline & Marasmius spp. & $\begin{array}{l}\text { Aya ala cari } \\
\text { Chincha ala } \\
\text { Micuna ala } \\
\text { Sara ala }\end{array}$ & $\begin{array}{l}\text { Man's spirit mushroom } \\
\text { Soft mushroom } \\
\text { Edible mushroom } \\
\text { Corn's mushroom }\end{array}$ & Litter & No \\
\hline & Mycena spp. & $\begin{array}{l}\text { Chonta ala } \\
\text { Shiquitu ala }\end{array}$ & $\begin{array}{l}\text { Chonta's mushroom } \\
\text { Tiny mushroom }\end{array}$ & Wood & No \\
\hline & $\begin{array}{l}\text { Oudemansiella cubensis (Berk. } \\
\text { \& M.A. Curtis) R.H. Petersen }\end{array}$ & Chincha ala & Soft, edible mushroom & Wood & No \\
\hline & Phellinus gilvus (Schwein.) Pat. & Aya ala & Ghost's mushroom & Wood & No \\
\hline & $\begin{array}{l}\text { Pleurotus concavus (Berk.) } \\
\text { Singer }\end{array}$ & Taka ala & Trunk's mushroom & Wood & No \\
\hline & Polyporus arcularius Rostk. & $\begin{array}{l}\text { Urpi ala } \\
\text { Sara ala }\end{array}$ & $\begin{array}{l}\text { Partridge's mushroom } \\
\text { Corn's mushroom }\end{array}$ & Wood & No \\
\hline & Polyporus spp. & $\begin{array}{l}\text { Aya ala } \\
\text { Urpi ala }\end{array}$ & $\begin{array}{l}\text { Ghost's mushroom } \\
\text { Partridge's mushroom }\end{array}$ & Wood & No \\
\hline & $\begin{array}{l}\text { Rigidoporus amazonicus } \\
\text { Ryvarden }\end{array}$ & Atun ala & Tuna's mushroom & Wood & No \\
\hline & Schizophyllum commune Fr. & Aya ala & Ghost's mushroom & Wood & No \\
\hline & $\begin{array}{l}\text { Scutellinia scutellata (L.) } \\
\text { Lambotte }\end{array}$ & Ringri ala & Ear's mushroom & Wood & No \\
\hline & Thelephora sp. & Aya ala & Ghost's mushroom & Soil & No \\
\hline & Trametes versicolor (L.) Lloyd & $\begin{array}{l}\text { Chincha ala } \\
\text { Ilma ala } \\
\text { Luciru ala } \\
\text { Sara ala }\end{array}$ & $\begin{array}{l}\text { Edible mushroom } \\
\text { Soft mushroom } \\
\text { Star mushroom } \\
\text { Corn's mushroom }\end{array}$ & Wood & No \\
\hline & Tremella fuciformis Berk. & Calun ala & Jelly mushroom & Wood & No \\
\hline & $\begin{array}{l}\text { Volvariella volvacea (Bull.) } \\
\text { Singer }\end{array}$ & Palanda ala & Banana's mushroom & $\begin{array}{l}\text { Banana's } \\
\text { leaves }\end{array}$ & No \\
\hline Secoyas/ Pai'coca /Amazonia & $\begin{array}{l}\text { Auricularia delicata (Mont.) } \\
\text { Henn. }\end{array}$ & Caro tëti & $\begin{array}{l}\text { Mushroom in shape of a } \\
\text { plate }\end{array}$ & Wood & No \\
\hline & $\begin{array}{l}\text { Auricularia fuscosuccinea } \\
\text { (Mont.) Henn. }\end{array}$ & Cajoro tëti & Ear mushroom & Wood & No \\
\hline & Favolus tenuiculus P.Beauv. & Ai tëti & Edible mushroom & Wood & No \\
\hline
\end{tabular}

Published: 12 December 2019

http://dx.doi.org/10.32859/era.18.38.1-15 


\section{Sionas/ Bai'coca /Amazonia}

Shuar/ Shuar Chicham/Amazonia

\begin{tabular}{|c|c|c|c|c|}
\hline & $\begin{array}{l}\text { Cajo tëti } \\
\text { Mawue tëti }\end{array}$ & $\begin{array}{l}\text { Ear mushroom } \\
\text { Edible mushroom }\end{array}$ & & \\
\hline $\begin{array}{l}\text { Marasmiellus cf. albidus (Murrill) } \\
\text { Singer }\end{array}$ & Imi tëti & Sardine's mushroom & Litter/Wood & No \\
\hline Monilia sp. & A'so pore & Yucca's dust & Cassava & No \\
\hline $\begin{array}{l}\text { Oudemansiella cubensis (Berk. } \\
\text { \& M.A. Curtis) R.H. Petersen }\end{array}$ & Imi tëti & Sardine's mushroom & Wood & No \\
\hline $\begin{array}{l}\text { Pleurotus djamor (Rumph. ex } \\
\text { Fr.) Boedijn }\end{array}$ & Po tëti & White mushroom & Wood & No \\
\hline Schizophyllum commune Fr. & Tëti & Mushroom & Wood & No \\
\hline $\begin{array}{l}\text { Auricularia delicata (Mont.) } \\
\text { Henn. }\end{array}$ & Cajo tëti & Ear mushroom & Wood & No \\
\hline $\begin{array}{l}\text { Auricularia fuscosuccinea } \\
\text { (Mont.) Henn. }\end{array}$ & Cajoro tëti & Ear mushroom & Wood & No \\
\hline Favolus tenuiculus P.Beauv. & Ai tëti & Edible mushroom & Wood & No \\
\hline Lentinus sajor-caju (Fr.) Fr. & Ai tëti & Edible mushroom & Wood & No \\
\hline $\begin{array}{l}\text { Oudemansiella cubensis (Berk. } \\
\text { \& M.A. Curtis) R.H. Petersen }\end{array}$ & Imi tëti & Sardine's mushroom & Wood & No \\
\hline $\begin{array}{l}\text { Pleurotus djamor (Rumph. Ex } \\
\text { Fr.) Boedijn }\end{array}$ & Po tëti & Whitish mushroom & Wood & No \\
\hline Schizophyllum commune Fr. & Tëti & Mushroom & Wood & No \\
\hline $\begin{array}{l}\text { Auricularia delicata (Mont.) } \\
\text { Henn. }\end{array}$ & Iwianchi kuishi & Devil's ear & Wood & No \\
\hline $\begin{array}{l}\text { Auricularia fuscosuccinea } \\
\text { (Mont.) Henn. }\end{array}$ & Iwianchi kuishi & Devil's ear & Wood & No \\
\hline $\begin{array}{l}\text { Clavulinopsis fusiformis } \\
\text { (Sowerby) Corner }\end{array}$ & Sapi & Worm's mushroom & Soil & No \\
\hline $\begin{array}{l}\text { Collybia cf.hirtella (Berk. \& } \\
\text { Broome) Dennis }\end{array}$ & Iwianchi kuishi & Devil's mushroom & Wood & No \\
\hline $\begin{array}{l}\text { Ophiocordyceps melolonthae } \\
\text { (Tul. \& C. Tul.) G.H. Sung, J.M. } \\
\text { Sung, Hywel-Jones \& Stapafora }\end{array}$ & Sapi & Worm & Ant & No \\
\hline $\begin{array}{l}\text { Cotylidia aurantiaca (Pers.) } \\
\text { A.L.Welden }\end{array}$ & Mukuch & Mushroom & Wood & No \\
\hline Favolus tenuiculus P.Beauv. & Shuishui esemp & Edible mushroom & Wood & No \\
\hline
\end{tabular}

Published: 12 December 2019

http://dx.doi.org/10.32859/era.18.38.1-15 
Ethnobotany Research and Applications

\begin{tabular}{|c|c|c|c|c|c|}
\hline & Lentinus sajor-caju (Fr.) Fr. & Esemp & Mushroom & Wood & No \\
\hline & Polyporus tricholoma Mont. & Esemp & Mushroom & Wood & No \\
\hline \multirow{6}{*}{$\begin{array}{l}\text { Shiwiar/ Shiwiar chicham } \\
\text { /Amazonia }\end{array}$} & $\begin{array}{l}\text { Auricularia delicata (Mont.) } \\
\text { Henn. }\end{array}$ & Iwianchi kuishi & Devil's ear & Wood & No \\
\hline & $\begin{array}{l}\text { Auricularia fuscosuccinea } \\
\text { (Mont.) Henn. }\end{array}$ & Iwianchi kuishi & Devil's ear & Wood & No \\
\hline & $\begin{array}{l}\text { Clavulinopsis fusiformis } \\
\text { (Sowerby) Corner }\end{array}$ & Sapi & Worm & Soil & No \\
\hline & $\begin{array}{l}\text { Cotylidia aurantiaca (Pers.) } \\
\text { A.L.Welden }\end{array}$ & Mukuch & Mushroom & Wood & No \\
\hline & Favolus tenuiculus P.Beauv. & Esemp & Edible mushroom & Wood & No \\
\hline & Lentinus sajor-caju (Fr.) Fr. & Shushui esemp & Edible mushroom & Wood & No \\
\hline \multirow{2}{*}{$\begin{array}{l}\text { Cofanes/ A'ingae } \\
\text { / Amazonia }\end{array}$} & $\begin{array}{l}\text { Auricularia fuscosuccinea } \\
\text { (Mont.) Henn. }\end{array}$ & Anjse tsina & Reddish mushroom & Wood & No \\
\hline & Lentinus sajor-caju (Fr.) Fr. & Toto'a tsina & Whitish mushroom & Wood & No \\
\hline \multirow[t]{7}{*}{ Zaparas/ Zapara/Amazonia } & $\begin{array}{l}\text { Auricularia delicata (Mont.) } \\
\text { Henn. }\end{array}$ & Kalulu ala katsapija & Soft mushroom & Wood & No \\
\hline & Favolus tenuiculus P.Beauv. & San yaku katsapija & Edible mushroom & Wood & No \\
\hline & Gymnopilus cf. lepidotus Hesler & $\begin{array}{l}\text { Kiauka uwinjia } \\
\text { katsapija }\end{array}$ & Toucan's heart & Wood & No \\
\hline & Lentinus crinitus (L.) Fr. & Matsakau katsaija & Partridge's mushroom & Wood & No \\
\hline & Lycoperdon pyriforme Schaeff. & Kuinja katsapija & Mushroom in shape of a ball & Wood & No \\
\hline & $\begin{array}{l}\text { Oudemansiella cubensis (Berk. } \\
\text { \& M.A. Curtis) R.H. Petersen }\end{array}$ & Chuchu katsapija & Raft's mushroom & Wood & No \\
\hline & $\begin{array}{l}\text { Pleurotus concavus (Berk.) } \\
\text { Singer }\end{array}$ & Aunika katsapija & Cigarette mushroom & Wood & No \\
\hline
\end{tabular}


Favolus tenuiculus is also eaten by the Patamona in Guyana (Henkel et al. 2004). The Sanama fry or cook this species (Fidalgo and Prance1976). RuánSoto et al. (2006) also cited the use of $F$. tenuiculus as food by Maya descendants.

Pleurotus djamor is cooked and eaten by the Sanama (Fidalgo and Prance 1976). Unidentified taxa of Pleurotus are also referred as edible by the Sanama (Fidalgo and Prance 1976), the Temuans (Chang and Lee 2004) and the Patamona (Henkel et al. 2004). Ruán-Soto et al. (2006) also referred to $P$. concavus and $P$. djamor as edible in Mexico.

Besides the most common species, Lentinus crinitus is used by the Sanama (Fidalgo and Prance 1976) and by the Patamona (Henkel et al. 2004). Other species of this genus were reported as edible by the Temuans (Chang and Lee 2004) and indigenous groups of Burkina Faso (Guissou et al. 2008). Polyporus tricholoma is eaten after cooking by the Tsachilas, as well as by the Sanama (Fidalgo and Prance 1976).

Schizophyllum commune is edible specie for the indigenous people in Malaysia (Chang and Li 2004), Nigeria (Akpaja et al. 2005) and Mexico (Ruán-Soto et al. 2006). In Ecuador, the initial stages of basidiomata are also used to restore energy to women who have recently given birth.

Gymnopus nubicola is consumed by the Kichwas in the Ecuadorian Andes. This species was taxonomicaly described by Halling (1996), and was for the first time reported as an edible, being known by the traditional name kallamba (Gamboa-Trujillo et al. 2014).

\section{Myths}

Ecuador contains 13 ethnic groups, but only three have myths and legends about edible mushrooms consumed by them:the mountain and the Amazonian Kichwas, and the highlands Shuars.

The mountain Kichwas call Agaricus pampeanus and $A$. argyropotamicus as kallambas de finados (Table 1) due to the occurrence and consumption of basidiomata in the period of the Day of the Dead. One interviewee also informed that these species represent food sent by God. The presence of their basidiomata also indicates the beginning of the winter and thunderstorm periods (Gamboa-Trujillo 2005; Gamboa-Trujillo et al. 2014). Lowy (1974) already observed that rain and thunderstorms are frequently related to the occurrence of Amanita muscaria (L.) Lam. in Guatemala and Mexico, and differently from the two Agaricus mentioned above, it is a poisonous species (Michelot \& Melendez-Howell 2003). The mountain Kichwas also believe that lightning causes the growth of the basidiomata. After this phenomenon, they harvest large quantity of these Agaricus species for household consumption, selling or exchanging by others traditional products in public markets of Sangolqui, Cantón Rumiñahui, Pichincha Province (Fig. 5). Thus, this is first report of informal selling of $A$. pampeanus on public markets (Fig. 6A-B). Montoya et al. (2004) and Pérez-Moreno et al. (2008) also reported the selling of species of Agaricus ( $A$. pampeanus and $A$. campestris) in Mexico.

The Amazonian Kichwas consider the emergence of Pleurotus concavus and Lentinus crinitus as indicators for early planting of Zea mays L. (corn, maiz). When these fungi emerge a lot during the winter, from November to April, the indigenous communities think that it is the ideal time to plant corn.

Finally, the older people of the communities relate stories about mushrooms and spirits forest in the highland 'paramos', and all-natural phenomena occurring with them. The Shuars name given to Auricularia fuscosuccinea, for example, is iwianchi kuishi that means "devil's ear". Also, the Secoyas frequently narrate stories about nocturnal wandering spirits in the forest during consumption of Geastrum australe (Piyaguaje 1990; Gamboa-Trujillo et al. 2005).

\section{Implication for conservation}

The three main landscape regions in Ecuador are definitely the Coast, Andes/Sierra and Amazon/Amazonia, and many different vegetation types can be found in each region (Sierra et al. 2002). As observed here, the richness of fungal species in the country used for humans is relatively high. However, $42 \%$ of all-natural area was already converted to any type of human-base land-use, representing a high loss of ecosystems (Sierra et al. 2002). Due to ecosystem loss, the fungi are also endangered, including some species that have typelocality in Ecuador. Gymnopus nubicola for example, on which the type locality is at 'páramos' (Halling 1996), is used as food resource (Gamboa-Trujillo et al. 2014). Unfortunately, part of this ecosystem was introduced by exotic grass and has been used for extensive agriculture (White and Maldonado 1991). In this region some species critical for extinction risks were referred (Sierra et al. 2002). Thus, actions on biodiversity conservation in Ecuador are urgent (Cuesta et al. 2017), including wild edible mushrooms. 


\section{Conclusions}

This research added many new ethnomycological data and improved the knowledge about the mycophagous behavior of many ethnic groups in Ecuador. There are about more than 1000 edible fungal species (Boa 2004) and 37 from Ecuador, a highly mycophilic (see Yasmin-Pasternak 2011 for summary) country. These studies must continue in order to prevent ethnobiological loss mostly caused by degradation of the environment and loss of the biodiversity in the regions where these people live (Tapia-Armijos et al. 2015). In addition, biological conservation of these ecosystems is urgent since many natural resources are used for these indigenous communities as food consumptions.

\section{Declarations:}

List of abbreviations:

QAP: Herbario Alfredo Paredes (Escuela de Biología, Universidad Central do Equador, Quito) URM:H erbário Pe. Camille Torrend (Departamento de Micologia, Universidade Federal de Pernambuco)

SENESCYT:S ecretaría de Educación Superior, Ciencia, Tecnología e Innovación del Ecuador CAPES: Coordenação de Aperfeiçoamento de Pessoal de Nível Superior

Ethics approval and consent to participate:A II participants gave their prior consent knowing the reason for the study, on which the interviews were carried out anonymously.

Consent for publication: Not applicable.

Availability of data and materials: No datasets have been deposited in public repositories.

Competing interest: Not applicable.

Funding: SENESCYT (Ecuador) and CAPES (Brazil) had important role in providing grant to PGT, as well financial support for his collection trips during these studies.

Author contributions: PGT designed the study, developed and review this manuscript; FW collaborated in the development and revision of the manuscript; TBG collaborated in the development and revision of the manuscript, and coordinated the research; CCM collaborated and coordinated the research; DA, PU, GG, ME, EC, JC, JP, NP, DP, VL, VL, MM, MC, MJ, SDLC, MC, SF, SA, CA, MDA, AM, $Y M$ and GF collaborated in many information reported in the manuscript.

\section{Acknowledgements}

The authors thank the SENESCYT (Ecuador) and CAPES (Brazil), for their scholarship, the Universidad Central del Ecuador for laboratory uses and facilities, Leonor Costa Maia for suggestions, and Stefan Brück and Sandra Páez, for the English improvements.

\section{Literature cited}

Adhikari MK, Devkota S, Tiwari RD. 2005. Ethnomycological knowledge on uses of wild mushrooms in Western and central Nepal. Our Nature 3:13-19.

Akpaja EO, Okhuoya JA, Ehwerheferere BA. 2005. Ethnomycology and uses of mushrooms among the Bini-speaking people of Nigeria:a case study of Aihuobabekun community near Benin City, Nigeria. International Journal of Medicinal Mushrooms 7:373374.

Boa E. 2004. Wild edible fungi. A Global overview of their use and importance to people. Food and Agriculture Organizzation of the United Nations, Rome, ITA.

Cardoso DBOS, Queiroz LP, Bandeira FP, GóesNeto A. 2010. Correlations between indigenous Brazilian folk classifications of fungi and their systematic. Journal of Ethnobiology 30:252-264.

Cerón CE, Montalvo C, Reyes C, Andi D. 2005. Etnobotánica Quichua Limoncocha, Sucumbios, Ecuador. Cinchonia 6:1-110.

Chang YS, Lee SS, 2004. Usefulness of macrofungi species in Malaysia. Fungal Diversity 15:15-22.

Corner EJH. 1950. A Monograph of Clavaria and allied genera. Oxford University Press, Cambridge, UK.

Cuesta F, Peralvo M, Merino-Viteri A, Bustamante M, Baquero F, Freile JF, Muriel P, Torres-Carvajal. 2017. Priority areas for biodiversity coeservation in mainland Ecuador. Neotropical Biodiversity 3:93106.

Denison W. 1967. Central American Pezizales II. The genus Cookeina. Mycologia 59:306-317.

Dennis RWG. 1956. Some Xylarias of tropical America. Kew Bulletin 11:401-444.

Fidalgo O. 1965. Conhecimento micológico dos índios brasileiros. Rickia 2:1-10.

Fidalgo O. 1985. A história da Micologia Brasileira:I. Brasil Colônia. Revista Brasileira da História da Ciência 2:47-51.

Fidalgo O, Prance GT. 1976. The ethnomycology of the Sanama indians. Mycologia 68:201-210. 
Gamboa-Trujillo JP. 2005. Diversidad y etnomicología de macromycetes, Cuenca Alta del Río Oglán, Pastaza, Ecuador. Cinchona 6:95-110.

Gamboa-Trujillo JP, Payaguaje D, Payaguaje J, Payaguaje A, Piyaguaje N. 2005. Introducción a la Etnomicología de la Comunidad Secoya:Copal, Águas Blancas, Reserva de Reproducción Faunística Cuyabeno, Sucumbios-Ecuador at Yepez P, de la Torre S, Cerón C, Palácios W. Al Início del Sendero:Estudios Etnobotánicos Secoyas. Ed. Arboleda, Quito. Pp. 63-70 and 97-99.

Gamboa-Trujillo JP, Wartchow F, Cerón C, Aules E, Aigaje C, Calvalcanti LH, Gibertoni TB. 2014. Traditional use of Gymnopus nubicola as food resource in a Kichwa community, Pichincha, Ecuador. Mycosphere 5:180-186.

Garibay-Orijel R, Cifuentes J, Estrada-Torres A, Caballero J. 2006. People using macro-fungal diversity in Oaxaca, Mexico. Fungal Diversity 21:4167.

Garibay-Orijel R, Caballero J, A. Estrada-Torres, Cifuentes J. 2007. Understanding cultural significance, the edible mushrooms case. Journal of Ethnobiology and Ethnomedicine 3:4.

Guissou KML, Lykke AM, Sankara P, Guinko S. 2008. Declining wild mushroom recognition and usage in Burkina Faso. Economic Botany 62:530539.

Guzmán G. 1997. Identificación de Hongos. Claves Dicotómicas. Editorial Limusa, México D.F.

Halling RE. 1996. Notes on Collybia V. Gymnopus section Levipedes in tropical South America, with comments on Collybia. Brittonia 48:487-494.

Heinemann P. 1961. Agarici Austro-americani I:Agaricus of Trinidad. Kew Bulletin 15:231-248.

Henkel TW, Aime MC, Chin M, Andrew C. 2004. Edible mushrooms from Guyana. Mycologist 18, 104-108.

Henkel TW. 2005. Parakari, an indigenous fermented beverage using amylolytic Rhizopus in Guyana. Mycologia 97:1-11.

Iturriaga T, Pfister DH. 2006. A monograph of the genus Cookeina (Ascomycota, Pezizales, Sarcoscyphaceae). Mycotaxon 95:137-180.

Lowy B. 1974. Amanita muscaria and the thunderbolt legend in Guatemala and Mexico. Mycologia 66:188191.

Michelot D, Melendez-Howell LM. 2003. Amanita muscaria: chemistry, biology, toxicology, and ethnomycology. Mycological Research 107:131-146.

Morales O, Medel R, Guzmán G. 2006. Primer registro de la comestibilidad de una especie de Daldinia (Ascomycota). Revista Mexicana de Micologia 23:103-105.
Montoya A, Hernández-Totomoch O, Estrada-Torres A, Kong A, Caballero J. 2003. Traditional knowledge about mushrooms in a Nahua community in the state of Tlaxcala, México. Mycologia 95:793-806.

Montoya A, Kong A, Estrada-Torres A, Cifuentes J, Caballero J. 2004. Useful wild fungi of La Malinche National Park, Mexico. Fungal Diversity 17:115-143. Montoya A, Hernández N, Mapes C, Kong A, Estrada-Torres A. 2008. The collection and sale of Wild mushrooms in a community of Tlaxcala, Mexico. Economic Botany 62:413-424.

Pérez-Moreno J, Martínez-Reyes $M$, Yescas-Pérez A, Delgado-Alvarado A, Xoconostle-Cázeres B. 2008. Wild mushroom markets in Central Mexico and a case study at Ozumba. Economic Botany 62:425-436.

Payaguaje F. 1990. Bebedor de Yaje. Centro de Documentación de Sistema de Naciones Unidas, Quito, Ecuador.

Prance GT. 1973. The mycological diet of the Yanomam Indians. Mycologia 65:248-250.

Ruan-Soto F, Garibey-Orijel R, Cifuentes J. 2006. Process and dynamics of traditional selling wild edible mushrooms in tropical Mexico. Journal of Ethnobiology and Ethnomedicine 2:3.

Ryvarden L. 1991. Genera of Polypores. Nomenclature and taxonomy. Fungiflora, Oslo, Norway.

Singer R. 1986. The Agaricales in Modern Taxonomy. $4^{\text {th }}$ ed. Koeltz Scientific Books, Koegnistein, Germany.

Tapia-Armijos MF, Homeier J, Espinosa Cl, Leuschner C, de la Cruz M. 2015. Deforestatuion and forest fragmentation in South Ecuador since the 1970 s - losing a hotspot of Biodiversity. PLoS ONE 10:e0133701.

Vasco-Palacios AM, Suaza SC, Castaño-Betancur M, Franco-Molano AE. 2008. Conocimento etnoecológico de los hongos entre los indígenas Uitoto, Muiname y Andoke de la Amazonía Colombiana. Acta Amazonica 38:17-30.

Yasmin-Pasternak S. 2011. Ethnomycology:Fungi and Mushrooms in Cultural Entanglement at Anderson EN, Pearshall D, Hunn E, Turner N. Ethnobiology. John Wiley \& Sons, Inc., New Jersey. Pp 213-230.

Zent EL. 2008. Mushrooms for life among the Jotï in the Venezuelan Guayana. Economic Botany 63:471481.

Zent EL, Zent S, Iturriaga T. 2004. Knowledge and use of fungi by a mycophillic society of the Venezuelan Amazon. Economic Botany 58:214-226 


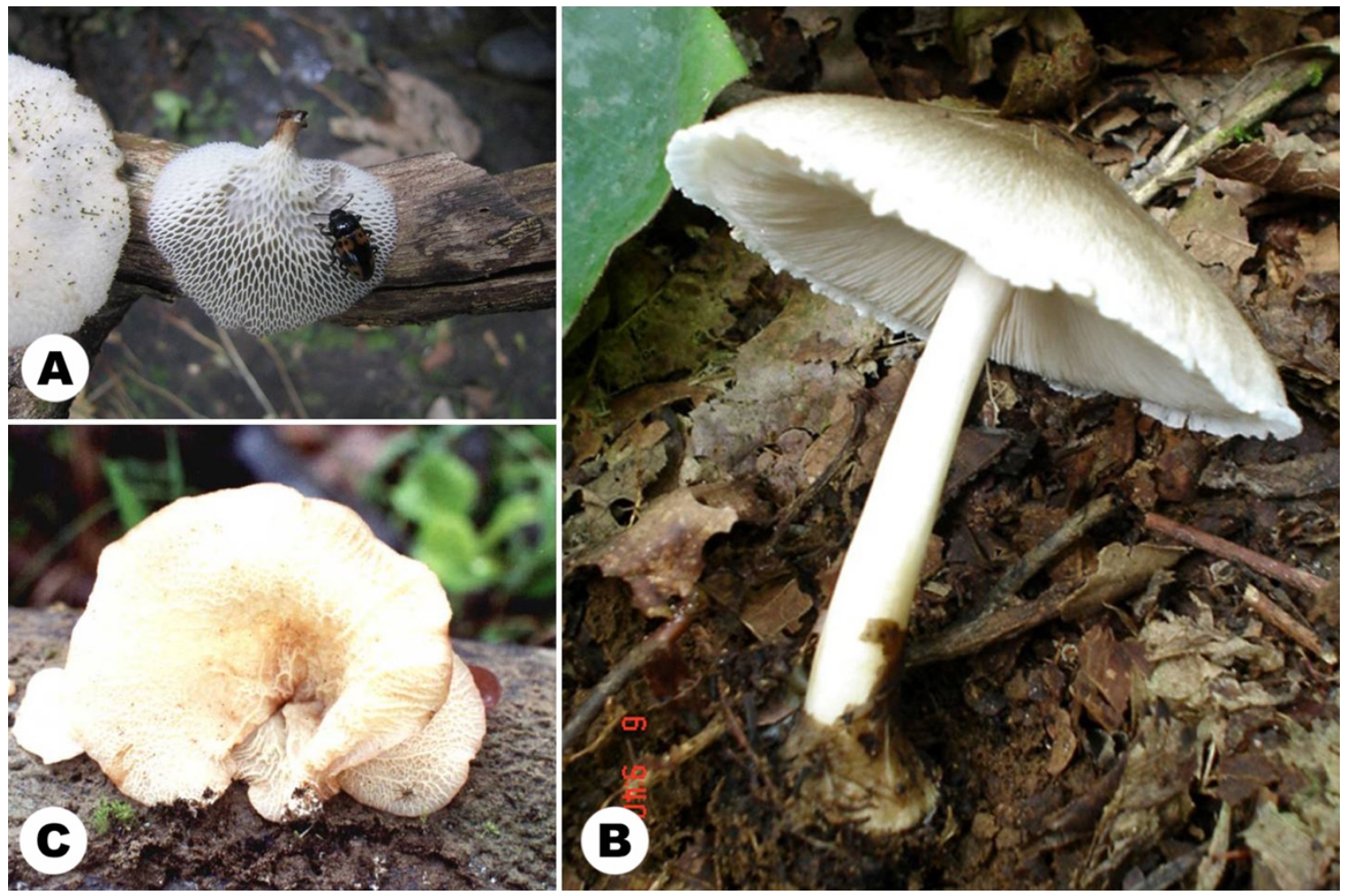

Figure 1. Edible mushrooms of Ecuador. A. Favolus tenuiculus. B. Volvariella volvacea. C. Auricularia delicata. Photos by J.P. Gamboa-Trujillo, all rights reserved.

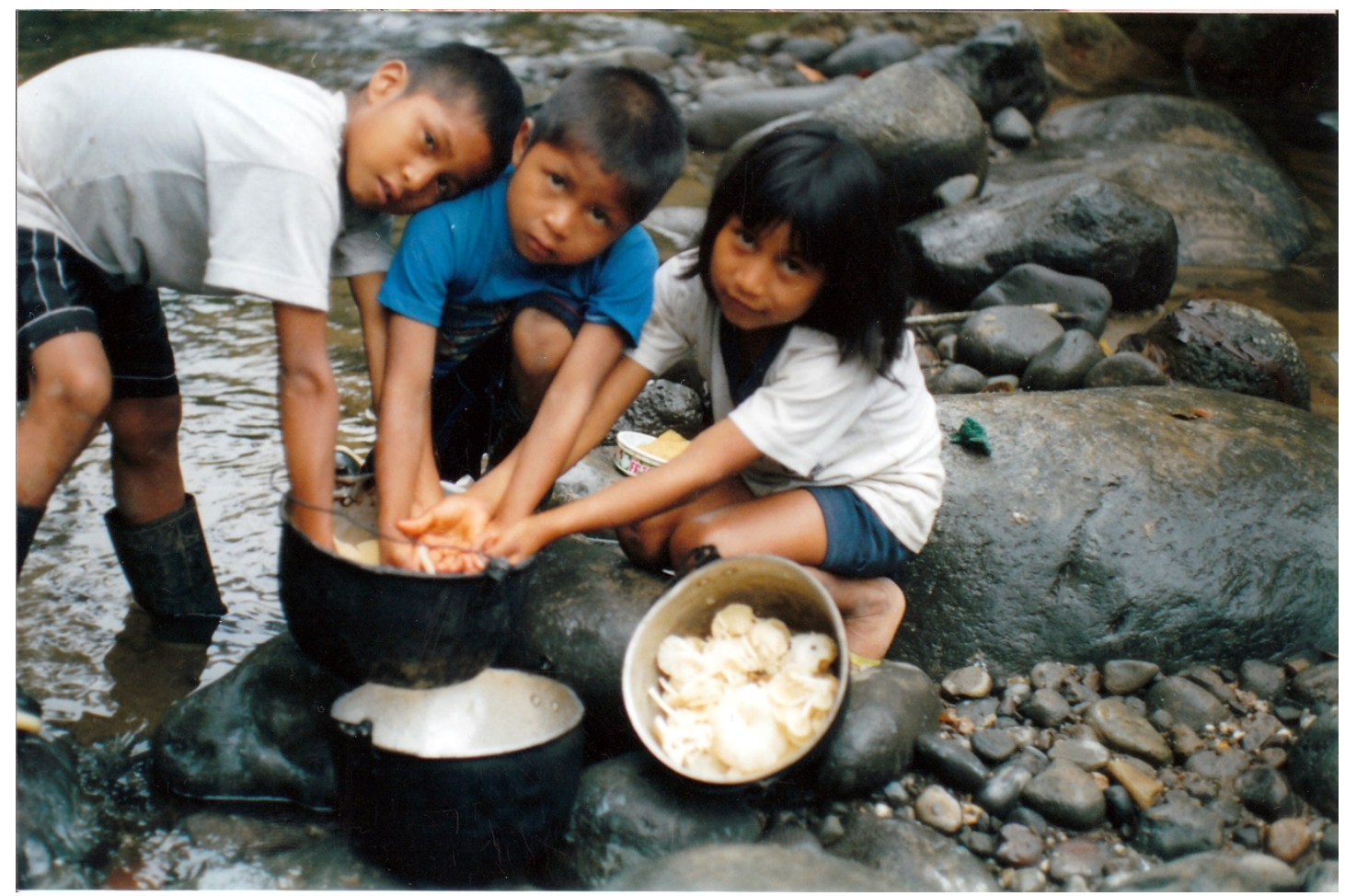

Figure 2. Mushrooms washed by children before cooking. Photo by J.P. Gamboa-Trujillo, all rights reserved. 

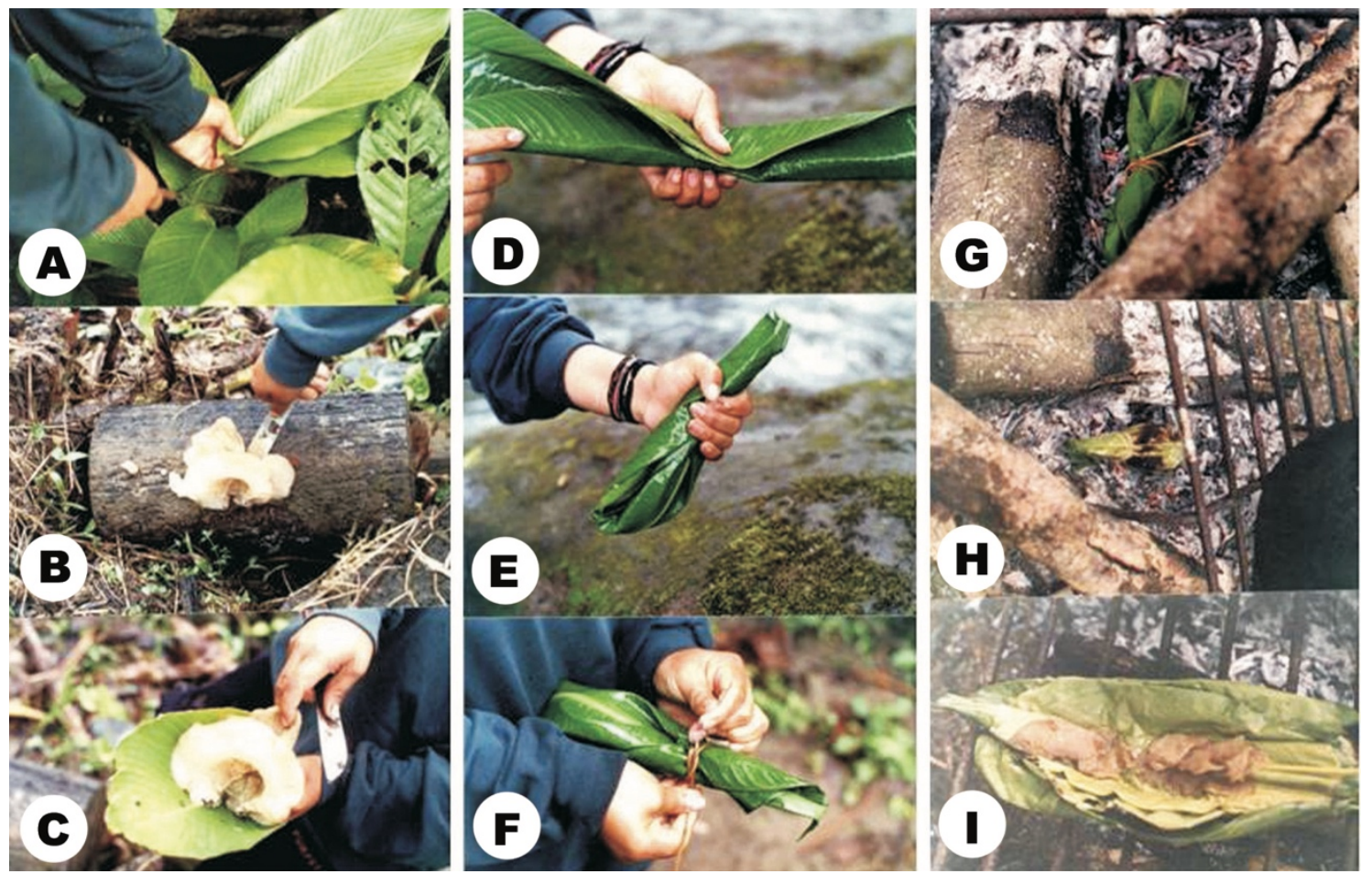

Figure 3. A-I. Traditional way to prepare mushrooms:Bundle of Auricularia delicata in leaves of Heliconia stricta "platanillo." Photos by J.P. Gamboa-Trujillo, all rights reserved.
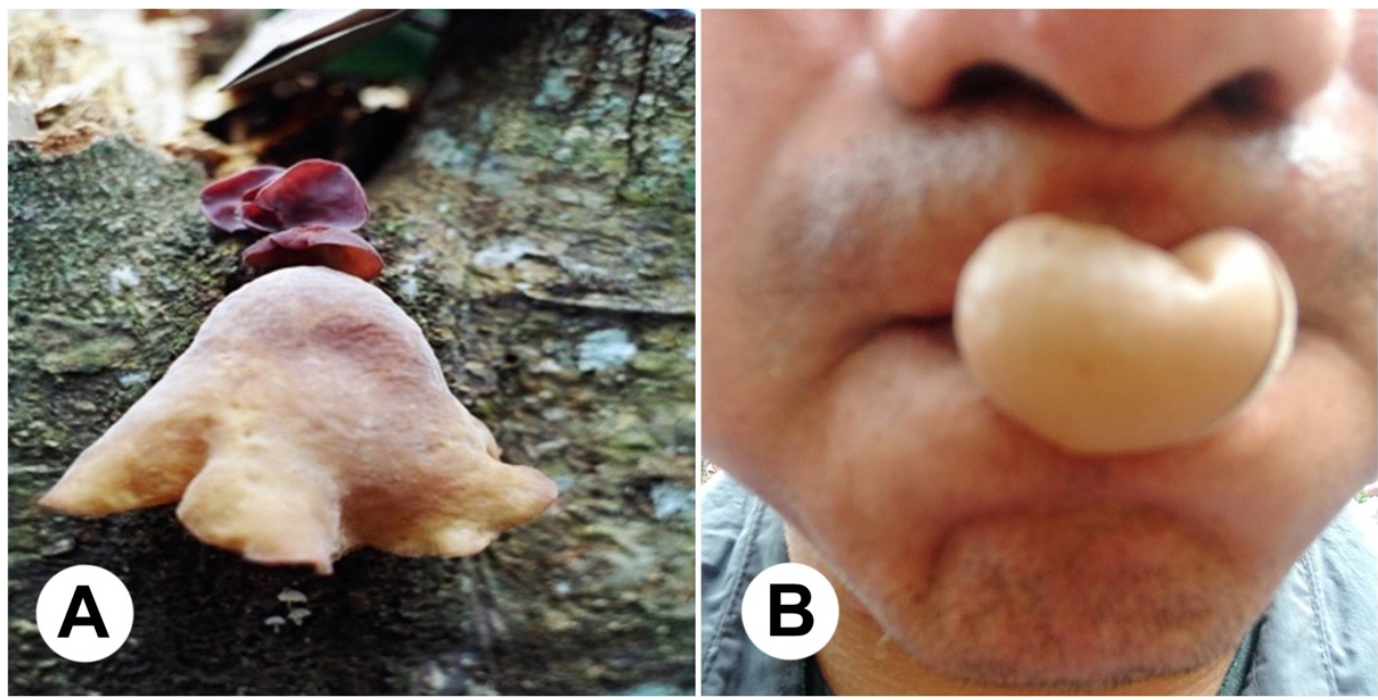

Figure 4. A. Basidiomata of Auricularia fuscosuccinea. B. Mushrooms of $A$. fuscosuccinea used as edible and toy for the childrens in Wapu Village, Morona Santiago province, Ecuador. Photo by J.P. Gamboa-Trujillo, all rights reserved. 


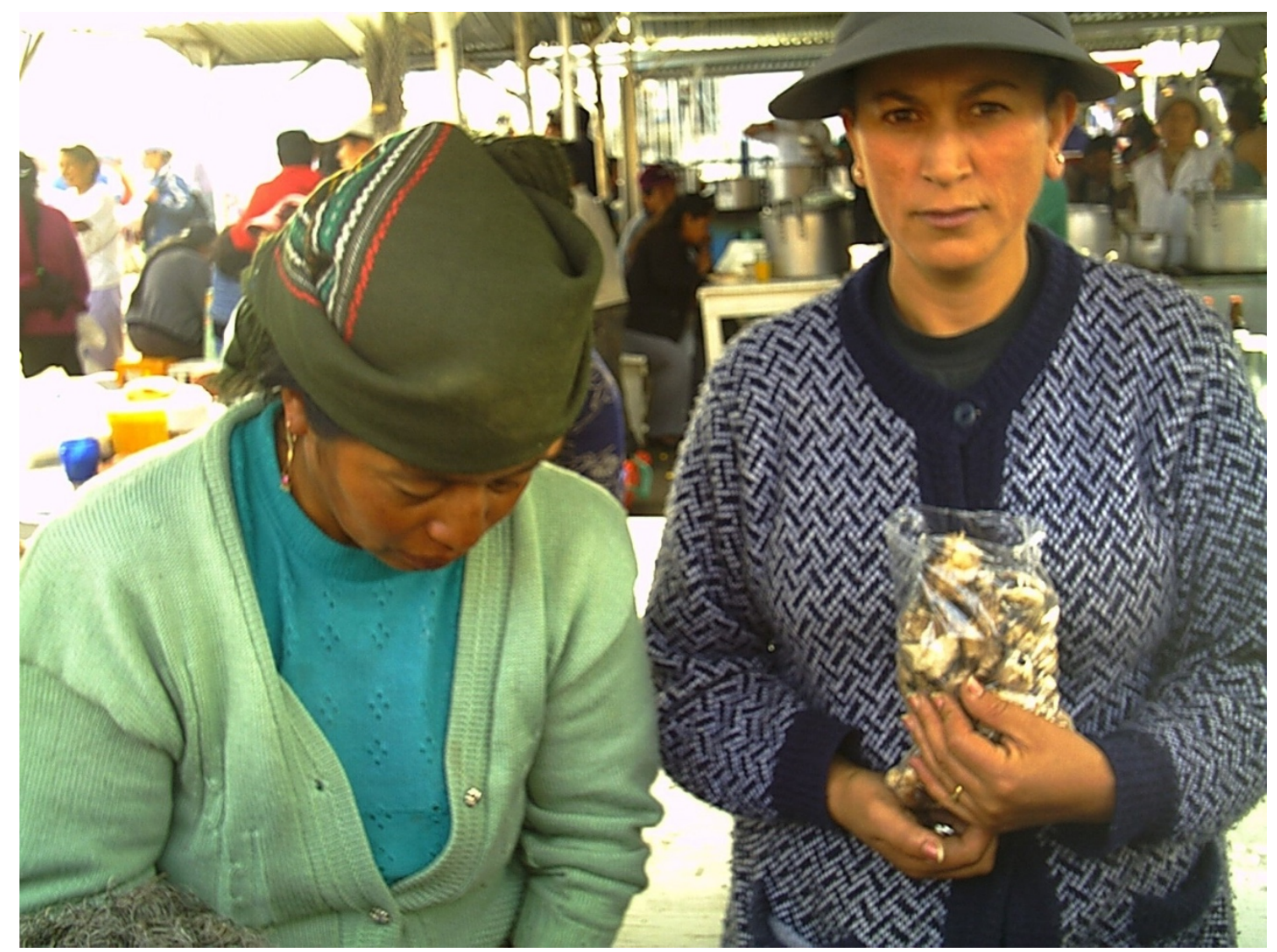

Figure 5. Selling of $A$. pampeanus and A. argyropotamicus (kallamba) on a traditional market in Sangolqui village, Pichincha province, Ecuador. Photo by J.P. Gamboa-Trujillo, all rights reserved. 

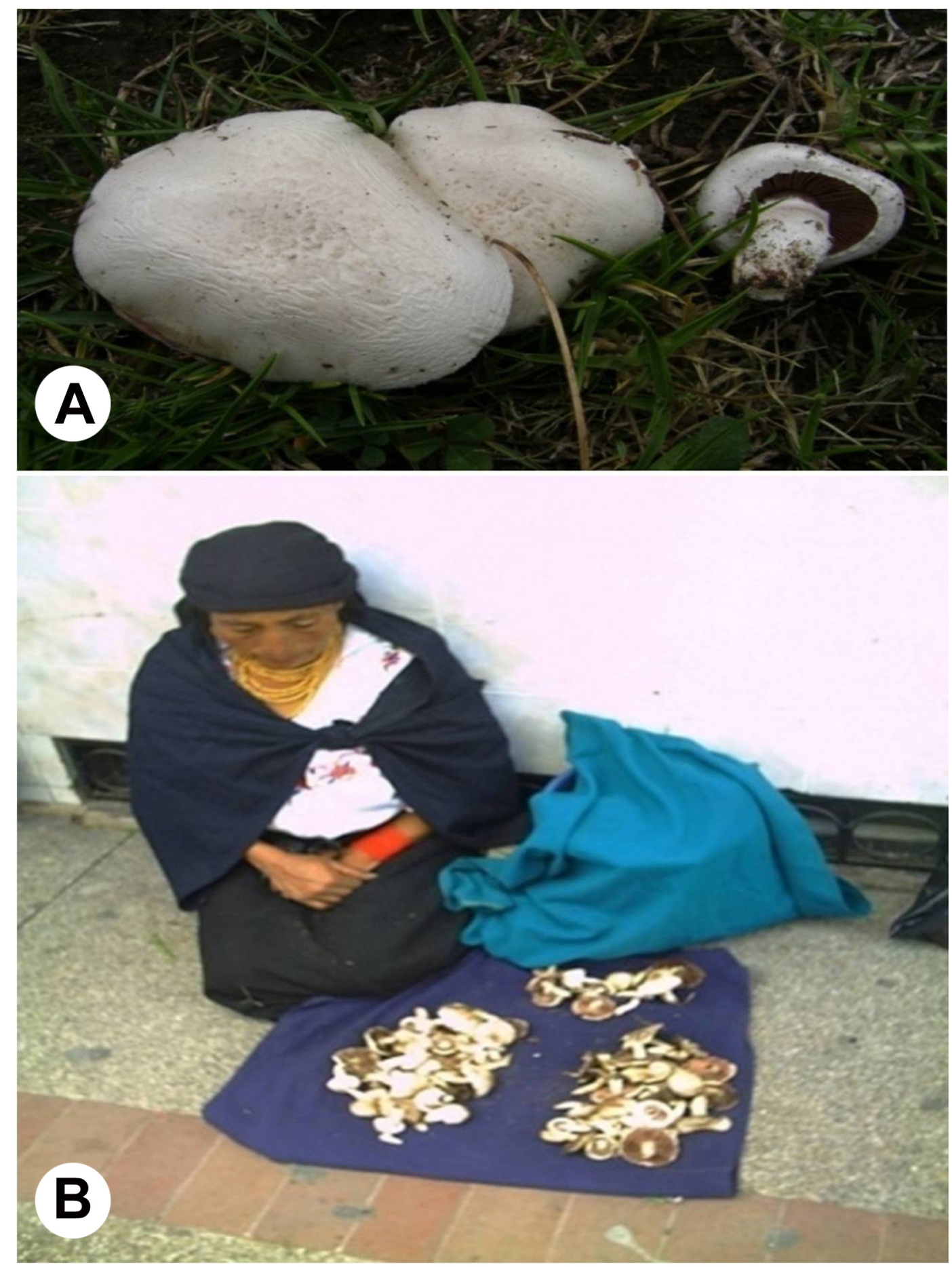

Figure 6. A. Basidiomata of Agaricus pampeanus. B. Woman selling of A. pampeanus (kallamba) on the sidewalk in Otavalo village, Imbabura province, Ecuador. Photo by J.P. Gamboa-Trujillo, all rights reserved. 